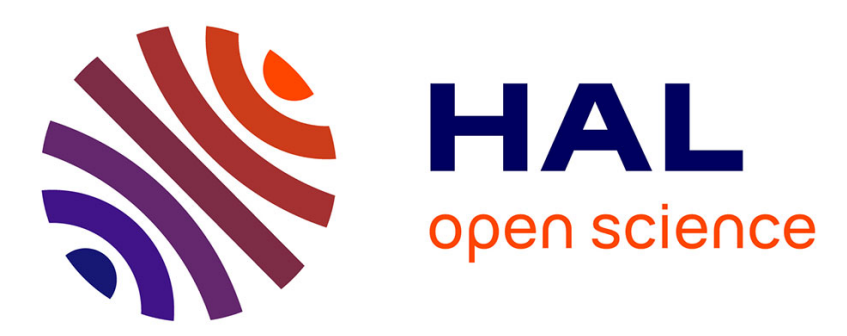

\title{
L'accès aux soins des étrangers en situation irrégulière en France: une prise en charge au titre de la pauvreté et de la compassion
}

Céline Gabarro

\section{- To cite this version:}

Céline Gabarro. L'accès aux soins des étrangers en situation irrégulière en France: une prise en charge au titre de la pauvreté et de la compassion. Migrations Société, 2018, №171 (1), pp.93-104. 10.3917/migra.171.0093 . hal-03263364

\section{HAL Id: hal-03263364 \\ https://hal.science/hal-03263364}

Submitted on 17 Jun 2021

HAL is a multi-disciplinary open access archive for the deposit and dissemination of scientific research documents, whether they are published or not. The documents may come from teaching and research institutions in France or abroad, or from public or private research centers.
L'archive ouverte pluridisciplinaire HAL, est destinée au dépôt et à la diffusion de documents scientifiques de niveau recherche, publiés ou non, émanant des établissements d'enseignement et de recherche français ou étrangers, des laboratoires publics ou privés. 


\title{
L'accès aux soins des étrangers en situation irrégulière en France : une prise en charge au titre de la pauvreté et de la compassion
}

\author{
Céline Gabarro, Docteure en sociologie, chercheure associée à l'Urmis
}

\section{[Version auteure]}

Référence : Gabarro Céline (2018). L'accès aux soins des étrangers en situation irrégulière en France : une prise en charge au titre de la pauvreté et de la compassion, Migrations société, 171(30), pp. 93-104. https://doi.org/10.3917/migra.171.0093

Le droit à la santé pour tous est garanti par plusieurs traités internationaux, comme le pacte international relatif aux droits économiques, sociaux et culturels des Nations Unies ou la Convention européenne des droits de l'homme. Selon ces textes, les étrangers, quel que soit leur statut juridique, doivent pouvoir jouir d'un accès aux soins. En outre, la santé est une composante du développement humain, d'autant plus que l'accès à une couverture maladie universelle constitue l'un des objectifs de développement durable des Nations Unies (ODD $\mathrm{n}^{\circ} 3^{1}$ ). Pour autant, les pays européens ne reconnaissent pas tous des droits sociaux aux étrangers en situation irrégulière : certains leur accordent des aides ponctuelles pour se faire soigner, mais aucun ne les affilie à leur système de santé au même titre que les autres assurés.

Il paraît alors judicieux de se demander comment ces pays gèrent les questions d'accès aux soins des étrangers en situation irrégulière. Dans cet article, nous allons nous intéresser au cas français. Cet exemple est intéressant dans la mesure où la France est le seul pays européen à offrir une couverture maladie gratuite et réservée aux étrangers en situation irrégulière : l'aide médicale d'État (AME). Contrairement à d'autres pays, les étrangers en situation irrégulière peuvent, en France, accéder gratuitement au système général de soins, bénéficier d'une couverture maladie et être pris en charge dans un hôpital comme par la médecine de ville. Leur accès aux soins et à la couverture est-il pour autant garanti dans les mêmes termes que pour les personnes résidant régulièrement en France ? Sont-ils pris en charge au même titre que les autres patients? Si tel n'est pas le cas, que signifie cette différentiation?

Certaines recherches ont déjà pointé les discriminations rencontrées par ces personnes, du fait notamment de leur mise à l'écart dans le dispositif particulier de $1^{\prime} \mathrm{AME}^{2}$. Dans cet article, nous allons nous intéresser plutôt au type de couverture maladie et de droits que fournit l'AME. Ce sont les conceptions de la solidarité et de la citoyenneté sociale, véhiculées par les dispositifs de prise en charge, ainsi que leurs effets sur l'accès à la couverture, qui vont particulièrement nous intéresser ici. Pour étudier cela, nous allons revenir sur l'évolution des

\footnotetext{
${ }^{1}$ L'objectif de développement durable $\mathrm{n}^{\circ} 3$ « Permettre à tous de vivre en bonne santé et promouvoir le bien-être de tous à tout âge » enjoint les pays dans son point 3.8 à «Faire en sorte que chacun bénéficie d'une couverture sanitaire universelle, comprenant une protection contre les risques financiers et donnant accès à des services de santé essentiels de qualité et à des médicaments et vaccins essentiels sûrs, efficaces, de qualité et d'un coût abordable ». Source : http://www.un.org/sustainabledevelopment/fr/health/

${ }^{2}$ CARDE, Estelle, “On ne laisse mourir personne'. Les discriminations dans l'accès aux soins”, Travailler, $\mathrm{n}^{\circ} 16,2006$, pp. 57-80.

GABARRO, Céline, "Les demandeurs de l'aide médicale d'État pris entre productivisme et gestion spécifique", Revue Européenne des Migrations Internationales, vol. 28, $\mathrm{n}^{\circ}$ 2, 2012, pp. 35-56.

IZAMBERT, Caroline, "Les limites de l'universel. L'accès des sans-papiers à une couverture maladie en France depuis 1999”, Les Cahiers du centre Georges Canguilhem, vol. 6, n 1, 2014, pp. 199-215.
} 
référentiels d'affiliation en matière de protection maladie, de 1945 jusqu'à la mise en place de l'aide médicale d'État au début des années 2000. Par "référentiel d'affiliation », nous entendons les moyens par lesquels, et les statuts au nom desquels, les personnes ont pu être rattachées à un système de protection maladie. En revenant sur la genèse de l'affiliation à la protection maladie des étrangers en situation irrégulière en France, nous verrons que nous sommes passés d'une prise en charge généraliste, pensée en termes de droit social, à une mise à l'écart et à des prises en charges au titre de la pauvreté et de la compassion, ce qui stigmatise le bénéficiaire et rend sa prise en charge moins légitime et plus sujette à débats.

Notre analyse s'appuie sur une longue enquête ethnographique sur l'attribution de l'AME des deux côtés du guichet de l'Assurance maladie ${ }^{3}$. Elle repose sur onze mois d'observation auprès des agents de trois services d'une Caisse primaire d'assurance maladie (CPAM) l'accueil, la réglementation et l'instruction des demandes d'AME - et l'accompagnement de plusieurs demandeurs dans une deuxième Caisse, ainsi que des entretiens formels avec une dizaine d'entre eux.

\section{Une affiliation aveugle à la condition administrative des étrangers}

Les travaux de Gérard Noiriel ont montré que l'État social français s'est bâti, à la fin du XIX ${ }^{\mathrm{e}}$ siècle, sur la base d'un « universel national » ${ }^{4}$. C'est-à-dire que le droit social a défini des règles générales, valables pour tous, mais que cet universalisme se limitait aux nationaux. Toutes les grandes lois d'assurance et d'assistance votées à cette période restreignent ainsi le droit aux prestations sociales aux seuls Français. C'était le cas notamment pour l'aide médicale gratuite (AMG), une aide sociale accordée aux plus pauvres ${ }^{5}$ pour accéder aux soins à partir de $1893^{6}$ et ancêtre de l'AME. Les étrangers ne pouvaient en bénéficier que si leur pays d'origine avait signé un traité de réciprocité garantissant les mêmes avantages aux Français résidant sur leur $\operatorname{sol}^{7}$. Au départ, ce ne sont donc pas les étrangers en situation irrégulière qui sont exclus de la solidarité collective du fait de leur statut administratif, mais l'ensemble des étrangers en tant que non nationaux.

Cet « universel national » perdure en partie dans le système de Sécurité sociale qui se met en place en 1945. Certaines prestations sont ainsi subordonnées à une condition de nationalité, comme l'allocation adulte handicapé $(\mathrm{AAH})$. D'autres sont soumises à la régularité du séjour $^{8}$, comme les allocations familiales ${ }^{9}$. Ces conditions ne concernent, néanmoins, pas la

\footnotetext{
${ }^{3}$ GABARRO, Céline, L'attribution de l'aide médicale d'État (AME) par les agents de l'Assurance maladie. Entre soupçon de fraude, figures de l'Étranger et injonctions gestionnaires, Thèse de doctorat en sociologie, Paris : Université Paris Diderot, 2017, 474 p.

${ }^{4}$ NOIRIEL, Gérard, État, nation et immigration, Paris : Éd. Gallimard, 2005 (1 ère éd. Belin, 2001), 590 p.

${ }^{5}$ Nous entendons ici la pauvreté au sens de Georg Simmel, c'est-à-dire comme des personnes qui ont besoin d'aide sociale pour vivre. Voir SIMMEL, Georg, Les pauvres, Paris : Presses Universitaires de France, 2011 ( 1 ère éd. 1907), $112 \mathrm{p}$.

${ }^{6} \mathrm{BEC}$, Colette, L'assistance en démocratie. Les politiques assistantielles dans la France des XIX ${ }^{e}$ et XX $X^{e}$ siècles, Paris : Éd. Belin, 1998, 254 p.

7 DEWHURST LEWIS, Mary, Les frontières de la République. Immigration et limites de l'universalisme en France, 1918-1940, Paris : Éd. Agone, 2010, 361 p.

ISIDRO, Lola, L'étranger et la protection sociale, Thèse de doctorat en droit, Paris : Université Paris OuestNanterre La Défense, 2015, 663 p.

${ }^{8}$ Loi n ${ }^{\circ} 46-1385$ du 22 août 1946.
} 
protection maladie. Le système français d'assurance maladie, instauré en 1945, a pour cibles principales le travailleur et sa famille. En effet, c'est au titre du travail, en cotisant, que ce dernier se protège lui et ses ayants-droits (conjoint et enfants). Ce système repose ainsi au départ sur deux référentiels d'affiliation : le travail et la famille. Le second est subsidiaire au premier et ne permet pas d'être affilié en son nom, mais uniquement en tant «qu'ayant droit ».

En 1945, aucune différence de traitement n'existe entre les Français et les étrangers en situation régulière ou irrégulière. La nationalité et le statut de séjour n'entrent pas en compte s'agissant de l'affiliation à l'assurance maladie. Seuls les principes de territorialité (la personne doit résider en France pour en bénéficier) et de cotisation (la personne doit cotiser par le biais de son travail, ou celui de son parent, pour être protégée) prévalent. Les Français et les étrangers bénéficient ainsi, à cette époque, de l'assurance maladie dans les mêmes conditions, qu'ils soient en situation régulière ou irrégulière. L'important est en effet d'avoir un emploi, ou d'être ayant droit d'un salarié.

Les personnes qui ne peuvent être rattachées à l'assurance maladie peuvent, quant à elles, bénéficier du système d'aide sociale à travers l'aide médicale gratuite (AMG). L'AMG s'adresse autant aux Français qu'aux étrangers en situation régulière ou irrégulière. Ses bénéficiaires sont identifiés en fonction de leur niveau de ressources, de leur besoin de soins et doivent justifier d'une résidence dans le département qui attribue l'aide, mais en aucun cas de la régularité de leur séjour.

De 1945 à 1993, les étrangers en situation irrégulière sont ainsi affiliés en fonction des mêmes conditions que l'ensemble de la population : leur statut professionnel, leur résidence ou leurs revenus. Ils peuvent dépendre de la Sécurité sociale, comme de l'Aide sociale, leur statut d' « irréguliers » n'entrant pas en compte dans cette répartition. Au cours de cette période, les modes et niveaux de protection peuvent différer en fonction de la profession des personnes, mais pas en fonction de la régularité de leur séjour ou de leur origine nationale.

\section{La déconnexion entre droits sociaux et travail salarié pour les étrangers en situation irrégulière}

La loi Pasqua ${ }^{10}$ du 24 août 1993 marque un tournant dans l'affiliation à la Sécurité sociale, en subordonnant l'obtention de l'ensemble des prestations sociales à une condition de régularité du séjour. Cette loi a deux conséquences pour les étrangers.

Tout d'abord, elle inverse, pour eux, l'ordre des priorités dans les logiques d'affiliation. Jusqu'en 1993, c'était le fait de travailler qui prévalait. Ce n'était que dans le cas où une personne n'était pas salariée, qu'elle pouvait être protégée au titre de sa famille ou d'une prestation sociale. Ces derniers modes de rattachement étaient subsidiaires. À partir de 1993, le travail ne permet plus d'affilier directement les étrangers. Ce n'est plus le fait de cotiser qui prévaut dans leur cas mais, d'abord, d'être en situation régulière en France. La condition de

\footnotetext{
${ }^{9}$ MATH, Antoine, "Droit à la santé des ressortissants communautaires vivant en France. Les difficultés d'accès à la protection maladie et aux soins", Hommes \& Migrations, ${ }^{\circ}$ 1282, novembre-décembre 2009, pp. 122-135; IZAMBERT, Caroline, “30 ans de régressions dans l'accès aux soins”, Plein Droit, vol. 86, n 3, 2010, pp. 5-9.

${ }^{10}$ Du nom du ministre de l'Intérieur de l'époque, Charles Pasqua.
} 
régularité se rajoute ainsi et devient le critère déterminant de rattachement, primant sur celui du travail.

Deuxièmement, avec la réforme Pasqua, c'est aussi le statut protecteur de l'emploi qui n'est plus reconnu aux étrangers en situation irrégulière. En effet, avec la création de la Sécurité sociale en 1945, un nouveau régime de protection des travailleurs a été instauré. Alors qu'auparavant, seuls les pauvres étaient pris en charge au nom de la charité avec l'Eglise puis de l'assistance publique avec les communes et l'Etat, ce sont les travailleurs et leur famille qui sont les cibles des politiques sociales de l'après-guerre. La Sécurité sociale confère une protection au travailleur contre ce qui est désormais présenté comme des « risques sociaux » (accident, maladie, vieillesse, charge de famille) et pourraient lui faire perdre sa force de travail. Or le travailleur n'a que cette dernière pour vivre. C'est ainsi une politique de prévention de la pauvreté qui est permise grâce à la naissance du statut de l'emploi ${ }^{11}$. Par son travail, le salarié cotise à un salaire indirect qu'il peut toucher en cas de besoin s'il perd son emploi (chômage) ou ne peut plus l'exercer pour des raisons d'âge (retraite) ou de santé (assurance maladie). C'est ainsi son statut de salarié qui cotise qui lui permet d'avoir des droits.

En subordonnant la Sécurité sociale à une condition de régularité, la loi Pasqua revient sur cet élément, en niant le statut de travailleur aux étrangers en situation irrégulière. Ces derniers continuent à cotiser, mais ne peuvent réclamer le bénéfice de leurs droits au chômage, à la retraite ou à l'assurance maladie tant qu'ils sont en situation irrégulière. La loi Pasqua contribue ainsi à distinguer la réalisation d'un travail, des droits que ce dernier peut conférer. Le travail déclaré ne permet dès lors plus aux étrangers en situation irrégulière de jouir de protections, même s'ils y ont cotisé au préalable.

\section{L'aide médicale d'État : une prise en charge distincte au nom de l'irrégularité}

Exclus de l'assurance maladie à partir de 1993, les étrangers en situation irrégulière ne peuvent désormais plus bénéficier que de l'aide médicale. Cette prestation d'aide sociale n'offre pas les mêmes droits que l'assurance maladie. Tout d'abord, elle est soumise à une condition de ressources : tout le monde ne peut donc en bénéficier. Ceux qui dépassent le plafond n'ont, dès lors, droit à aucune couverture. Deuxièmement, elle est subordonnée à une condition de résidence : seules les personnes qui justifient vivre en France depuis plus de trois ans peuvent accéder aux soins de ville, les autres ne peuvent se rendre qu'à l'hôpital, ce qui ne leur offre pas le même suivi médical, notamment en termes de soins de prévention.

La première forme d'aide médicale, l'AMG, est remplacée par l'aide médicale départementale (AMD) en 1992, dans le but de garantir une meilleure couverture ${ }^{12}$. Désormais, les demandeurs n'ont plus à justifier d'un « besoin de soins » pour en bénéficier. Le passage par une commission a été remplacé par un barème de ressources qui permet de qualifier les situations des personnes de manière moins arbitraire qu'auparavant. De plus, l'aide est désormais attribuée pour une durée d'un an (et non plus pour la durée des soins) et pour toute

\footnotetext{
${ }^{11}$ CASTEL, Robert, Les métamorphoses de l'État social. Une chronique du salariat, Paris : Éd. Gallimard, 2010 ( 1 ère éd. Fayart, 1995), 813 p.

${ }^{12}$ Loi de lutte contre la pauvreté et l'exclusion sociale et professionnelle du 29 juillet 1992.
} 
la famille ${ }^{13}$. La distinction entre aide médicale de ville et aide médicale hospitalière est par contre conservée.

L'aide médicale se rapproche ainsi du fonctionnement de l'assurance maladie : elle s'apparente à une couverture, car elle est préventive. L'AMD s'éloigne du modèle de prestation individuelle délivrée suite à l'étude d'une situation précise, où l'arbitraire est plus grand, pour se rapprocher de celui, plus sûr, d'un droit accordé en fonction du statut de la personne, qui est ici celui de l'« exclu », du «pauvre », et qui s'évalue selon le calcul de ses ressources.

Néanmoins, l'AMD étant une prestation d'aide sociale, elle est gérée par les départements. Le plafond de ressources, tout comme le montant attribué et le fonctionnement de l'aide, varient donc selon les lieux, ce qui engendre des inégalités territoriales ${ }^{14}$. Très critiqué par les associations, ce dispositif est à nouveau réformé par la loi du 27 juillet 1999, qui le remplace par deux nouvelles couvertures maladie : la couverture maladie universelle (CMU) et l'aide médicale d'État (AME).

Avec la CMU, c'est un nouveau référentiel d'affiliation qui se met en place. Désormais, c'est le fait de résider en France qui ouvre des droits à l'assurance maladie. La CMU reste néanmoins une couverture subsidiaire : ce n'est que lorsque la personne ne peut être rattachée par aucun autre moyen à l'assurance maladie (emploi, famille, prestations sociales) qu'elle peut en bénéficier. Le critère de résidence ne se substitue donc pas à celui du travail, mais s'y ajoute. Ce nouveau référentiel n'est, de plus, pas accessible à tous : dans la veine de la réforme de 1993, la CMU est subordonnée à une condition de régularité.

L'instauration de la CMU inaugure toutefois un changement symbolique important. La CMU ne permet pas, en effet, de couvrir une nouvelle catégorie de personnes : l'ensemble de la population résidant en France était déjà protégé avant $1999^{15}$, notamment via l'AMD. La nouveauté réside en réalité dans un changement pratique et symbolique : avant 2000, ceux qui ne pouvaient être rattachés à l'assurance maladie dépendaient de l'aide sociale par le biais de l'AMD. Avec la création de la CMU, ils sont désormais affiliés à la Sécurité sociale. Ces personnes intègrent ainsi le système de l'assurance maladie et deviennent des «assurés sociaux », mais seuls les Français et les étrangers en situation régulière accèdent à l'assurance maladie. Les étrangers en situation irrégulière restent, quant à eux, dépendants de l'Aide sociale à travers l'AME. Ses critères d'obtention sont, par contre, désormais les mêmes sur l'ensemble du territoire ${ }^{16}$. Dans la continuité de la réforme de l'AMD, la couverture est ouverte pour un an et les demandeurs n'ont plus à démontrer un besoin de soins pour l'obtenir. Ses bénéficiaires sont pris en charge à $100 \%$ du tarif de la Sécurité sociale. Lorsque des médecins les reçoivent, ils n'ont pas le droit de faire des dépassements d'honoraire, et sont obligés d'appliquer le tiers payant (les patients n'ont pas à faire l'avance des frais).

L'AME peut ainsi être considérée comme une avancée du point de vue de la couverture proposée : cette dernière est plus stable et offre aux personnes la possibilité de pouvoir se

\footnotetext{
${ }^{13}$ CARDE, Estelle, “'On ne laisse mourir personne'. Les discriminations dans l'accès aux soins”, art. cité. ${ }^{14}$ BOISGUERIN, Bénédicte, BONNARDEL, Christine, GISSOT, Claude et RUAULT Marie (2001). L'aide médicale départementale : bilan 1996-1999, Etudes et Résultats, 126, DREES, 12 p.

${ }^{15}$ FROTIÉE, Brigitte, "La réforme française de la Couverture maladie universelle, entre risques sociaux et assurance maladie", Lien Social et Politique-RIAC, $\mathrm{n}^{\circ}$ 55, printemps 2006, pp. 33-44.

${ }^{16}$ Les plafonds de ressources ne varient plus selon les départements.
} 
soigner sur la durée. L'AME fonctionne comme n'importe quelle autre couverture : les demandeurs peuvent se rendre chez n'importe quel médecin, que ce soit en médecine de ville ou à l'hôpital. L'AME n'est plus une aide ponctuelle, accordée arbitrairement, mais une véritable couverture maladie, accordée selon des critères à vérifier : justifier de son identité, d'une résidence stable de plus de trois mois sur le territoire, et ne pas dépasser le plafond de ressources fixé par la loi. Enfin, elle est octroyée par les agents de l'Assurance maladie.

L'AME peut néanmoins également être analysée comme un signe de régression du point de vue des droits des étrangers en situation irrégulière, et ce surtout à partir de 2004. En effet, en 2000, seule la CMU est soumise à une condition de résidence de plus de trois mois. Les étrangers et les Français qui ne remplissent pas ce critère peuvent, en attendant, bénéficier de l'AME, puisque cette dernière n'est, alors, subordonnée à aucune condition de stabilité de résidence. Le public de l'AME est encore mixte. Il peut être stigmatisé en tant que pauvre, mais pas en fonction de son origine ou de sa situation administrative. Cette situation change avec la loi de finances rectificatives votée fin 2003, qui subordonne l'AME à une condition de résidence stable et ininterrompue à partir du 2 janvier $2004^{17}$. Les Français et étrangers en situation régulière, qui résident en France depuis moins de trois mois, ne peuvent désormais plus y recourir. L'AME s'adresse dorénavant uniquement aux étrangers en situation irrégulière, et son bénéfice devient, dès lors, stigmatisant.

La mise en place de critères de régularité et de résidence pour la CMU et l'AME modifie ainsi le type de public de l'aide sociale. Alors que l'AMD s'adressait à l'ensemble des personnes pauvres, sans critère de nationalité et de régularité, le système distingue désormais d'un côté les Français et les étrangers en situation régulière qui dépendent de l'assurance maladie par le biais de la CMU, et de l'autre les étrangers en situation irrégulière qui se retrouvent seuls bénéficiaires de l'aide sociale en matière de couverture maladie, via l'AME. L'opposition entre aide sociale et Sécurité sociale, qui reposait jusqu'à présent sur un critère de contributivité par le biais du travail, est désormais réduite au critère de régularité. Le bénéfice de l'aide sociale, dans le cas de l'AME, devient alors un marqueur d'irrégularité, en plus de la pauvreté. Et l'irrégularité, une condition à remplir pour obtenir l'AME.

\section{Une affiliation au titre de la pauvreté qui engendre du soupçon et complexifie l'accès aux soins pour les étrangers en situation irrégulière}

À partir de 1993, c'est ainsi principalement au titre de la pauvreté et de leur résidence en France que les étrangers peuvent bénéficier de la solidarité nationale, et non plus au titre du droit social en tant que salariés et assurés sociaux. C'est leur statut de pauvre qui leur permet d'acquérir des droits, mais des droits dont l'obtention, soumise à un certain nombre de conditions, est limitée et rendue plus difficile. Comprendre que la prise en charge légale des étrangers en situation irrégulière n'est abordée et rendu possible qu'à travers le prisme de la pauvreté, permet d'analyser les relations qui peuvent se jouer au guichet et les soupçons qui pèsent sur ces personnes.

Le système français de protection maladie, en déconnectant les étrangers en situation irrégulière du statut de travailleur rend plus complexe, moins légitime et plus contestable leur affiliation à la couverture maladie. La prise en charge qu'ils obtiennent ne provient en effet

\footnotetext{
${ }^{17}$ Article $\mathrm{n}^{\circ} 97$ de la loi de finances rectificative pour 2003 votée le 30 décembre 2003.
} 
plus de leur labeur (via des cotisations), mais de la solidarité nationale. Dès lors, comme pour toute aide sociale, la question de leur légitimité à bénéficier de l'aide de la collectivité se pose selon deux termes : font-ils partie de cette communauté ? Méritent-ils cette aide (autrement dit : sont-ils de « bons pauvres ») ?

À travers notre enquête de terrain menée dans une CPAM, nous avons pu constater qu'en raison des moyens d'affiliation qui leur sont ouverts, les étrangers en situation irrégulière sont avant tout considérés comme des personnes «pauvres » par les agents de l'Assurance maladie. Lors des interactions et de l'étude des dossiers, ce stigmate de la pauvreté vient s'entremêler à celui de leur irrégularité. C'est ainsi à la fois en tant que pauvres et en tant qu'irréguliers qu'ils sont considérés comme légitimes ou illégitimes à obtenir l'AME, soit parce qu'ils sont vus comme de «mauvais pauvres » qui viennent profiter du système en tant que « touristes médicaux », soit parce qu'ils sont considérés comme de « bons pauvres » en tant que personnes qui ne sont pas autorisées légalement à travailler et ne peuvent donc s'en sortir par elles-mêmes. Dans les deux cas, le travail est l'élément manquant de leur biographie et de leur dossier. Pourtant, certains demandeurs déclarent gagner de l'argent grâce à quelques heures de travail, mais dans ces cas-là, ce dernier est considéré comme étant du travail au noir. Et certains agents, mal à l'aise avec l'illégalité de cette activité, leur conseillent de ne pas le déclarer, de peur que cela se retourne contre les demandeurs, qui seraient alors accusés de contrevenir à la loi.

Ce statut de pauvre peut, de plus, faire l'objet d'interrogations et de soupçons. Le bon demandeur étant un vrai pauvre, l'agent doit contrôler et vérifier sa situation économique, en scrutant les manières dont les usagers sont habillés, ou les biens qu'ils possèdent. Les ressources non déclarées des étrangers étant difficiles à prouver, les agents doivent remplir un document qui permet de les évaluer : «la fiche entretien AME». À partir de cette fiche, les agents doivent interroger les demandeurs sur leurs dépenses: combien payent-il mensuellement pour leur loyer? leur électricité ? leur nourriture, leurs habits ? etc. Le montant calculé est ensuite comparé aux ressources déclarées. S'il lui est supérieur, le demandeur peut être accusé de mentir et d'être au-dessus du plafond de ressources. Le fait que l'AME soit accordée sous critères de ressources génère ainsi des soupçons d'abus ou de fraude vis-à-vis de ces demandeurs, là où un système universaliste en engendre moins puisque la prestation est accordée à tous sans conditions. Subordonner l'accès aux soins des étrangers en situation irrégulière à une condition de ressources favorise ainsi une économie du soupçon.

\section{Le fonds de soins urgents et vitaux : une prise en charge au titre des raisons humanitaire et gestionnaire}

Les étrangers en situation irrégulière qui ne remplissent pas les conditions d'obtention de l'AME (c'est-à-dire celle de ressources et celle de résidence ininterrompue de plus de trois mois) peuvent être pris en charge ponctuellement, à condition que leur état de santé nécessite des soins urgents et/ou vitaux, par le fonds des soins urgents et vitaux (FSUV). Ce dispositif, créé par la loi de finances rectificative pour 2003, n'est pas un système de protection maladie, mais un mode de remboursement des soins fournis en urgence. Il permet à l'Assurance maladie de prendre en charge des frais engagés à l'hôpital par des personnes sans couverture 
maladie, et pour des soins urgents et vitaux uniquement ${ }^{18}$. Le FSUV vise ainsi à soutenir l'hôpital face au risque de créance irrécouvrable. Il intervient donc a posteriori (la personne a déjà été opérée) et ne prend en charge que les soins et les médicaments délivrés à l'hôpital. Le remboursement est ponctuel : il ne concerne qu'une opération précise ou une période d'hospitalisation déterminée ${ }^{19}$. Le FSUV ne permet ainsi aucun soin de prévention ou de suivi médical. Il ne s'adresse qu'aux étrangers en situation irrégulière.

Avec le FSUV, c'est au titre de la compassion que les personnes sont prises en charge : seuls les malades qui ont un besoin urgent et vital de soins sont remboursés. Les autres doivent payés eux-mêmes les soins entrepris ou à venir. Ce mode de prise en charge procède ainsi d'un fonctionnement typique du « gouvernement humanitaire », notion développée par Didier Fassin qu'il définit comme "le déploiement des sentiments moraux dans les politiques contemporaines $»^{20}$. Le gouvernement humanitaire est un mode de gouvernement, de gestion des événements, qui porte une attention particulière à la souffrance et au malheur. Il repose sur une économie morale dont la raison humanitaire est le cœur. Le FSUV relève ainsi d'une politique de la compassion : "c'est la sympathie ressentie devant l'infortune du prochain qui produit l'indignation morale susceptible de générer une action visant à la faire cesser $\gg^{21}$. Comme l'explique Didier Fassin, ce sont les sentiments moraux, c'est-à-dire «les émotions qui nous portent vers les malheurs des autres et nous font souhaiter les corriger ${ }^{22}$, qui gouvernent ces politiques. Dans ce type de dispositif, c'est ainsi au nom de la souffrance et du malheur des autres qu'on agit, plus qu'au nom de l'injustice et de l'égalité des droits. Les mesures mises en place ne sont pas pérennes, et servent à soulager des problèmes ponctuellement. On agit dans l'urgence, sur une situation précise à un instant précis. La situation de la personne n'est pas interrogée dans sa globalité.

Selon Didier Fassin, la raison humanitaire sur laquelle repose ce type de dispositif gouverne des «vies précaires", à savoir «des existences qui ne sont pas assurées mais sont octroyées par la prière, autrement dit qui ne sont pas définies dans l'absolu d'une condition, mais dans la relation à ceux qui ont sur elles un pouvoir $\gg^{23}$. C'est ainsi au nom de leur humanité et de leur souffrance que les soins des étrangers en situation irrégulière sont remboursés : le droit à la vie ne peut leur être refusé au nom de leur irrégularité. C'est l'universalité de leur humanité et de leur souffrance qui est ici mise en avant. Le FSUV a d'ailleurs été mis en place pour éviter que l'article 97 de la loi de finances rectificative pour 2003, qui instaure la condition de stabilité en matière d'AME, soit jugée inconstitutionnelle car allant à l'encontre des droits de

\footnotetext{
${ }^{18}$ L'accouchement et le suivi de la maternité sont ainsi pris en charge au titre des soins urgents et vitaux, tout comme le VIH-sida ou la tuberculose.

${ }^{19}$ On pourrait également voir dans la mise en place du FSUV une logique de santé publique, ce dernier permettant d'éviter la propagation d'épidémies. Néanmoins, seuls les étrangers en situation irrégulière sont pris en charge par le FSUV, ceux en situation régulière qui sont en France depuis moins de trois mois, n'ont droit à aucun dispositif de couverture, et ne peuvent être pris en charge que par les permanences d'accès aux soins de santé (PASS), qui ont un budget limité, ou les associations. C'est pourquoi nous ne préférons pas avancer d'explication par la santé publique, qui reste pour nous une hypothèse à creuser, mais que notre travail n'a pas pu démontrer.

${ }^{20}$ FASSIN, Didier, La raison humanitaire. Une histoire morale du temps présent, Paris : Éd. du Seuil/Gallimard, 2010, 358 p. (voir p. 8).

${ }^{21}$ Ibidem.

${ }^{22}$ Ibidem (voir p. 7).

${ }^{23}$ Ibidem (voir p. 12).
} 
l'homme et notamment du droit à la santé pour tous ${ }^{24}$. Le corps de l'étranger est ainsi, ici, accepté uniquement comme un corps souffrant. C'est seulement quand la souffrance est intenable qu'il est pris en charge : uniquement quand il y a urgence et que le pronostic vital est engagé. Ne pas les prendre en charge est supportable tant que cela n'attente pas à leur vie. On ne parle pas d'universalité, de justice, de droit ou d'égalité ici, mais bien de prise en charge ponctuelle, dans l'urgence sans suivi sur le long terme.

La prise en charge des étrangers en situation irrégulière dans le cadre du FSUV relève ainsi d'une gestion de l'urgence, au cas par cas, ponctuelle, qui permet de sauver des vies, mais qui n'instaure aucun droit. Nous sommes finalement dans une gestion humanitaire proche de la médecine prodiguée dans les camps humanitaires ${ }^{25}$ : le corps souffrant permet une compassion et une prise en charge, mais il n'accorde pas de droits ou de statut social.

\section{Conclusion}

À travers cette description des dispositifs de l'AME et du FSUV, nous pouvons constater que la prise en charge des étrangers en situation irrégulière en France, bien qu'existante et intégrée au système général en partie, ne repose pas sur (ni ne traduit) un droit à la santé accordé de manière universelle ou selon des critères sociaux et politiques. C'est seulement au titre de la pauvreté ou de la maladie qu'ils sont pris en charge. Le fait d'être reconnu en tant que «pauvre » leur assure néanmoins une véritable couverture maladie (avec suivi et soins de prévention), bien que celle-ci reste dépendante d'un système à part, l'aide sociale, ce qui peut engendrer des difficultés d'accès aux soins. À l'inverse, le statut de malade ne leur accorde qu'une prise en charge ponctuelle à l'hôpital. Le statut de pauvre leur assure ainsi une reconnaissance sociale et leur permet d'acquérir un statut de patient qui a des droits ${ }^{26}$, tandis que celui de malade les renvoie à une vie liminale, à une simple existence physique sans considération sociale.

Le fait que les étrangers en situation irrégulière ne se voient pas reconnaître le statut de travailleur détermine ainsi le type de couverture qui leur est proposée, et marque aussi leur expérience sociale de santé et leurs trajectoires de soins. C'est ainsi au titre de leur pauvreté que les parlementaires débattent des bonnes raisons de les prendre ou non en charge. Au titre de leur pauvreté qu'ils méritent la commisération, ou qu'ils suscitent la crainte. L'accès à une couverture maladie n'est dès lors plus discutée en termes de droit social dans les débats parlementaires, mais en termes de prise en charge humanitaire au titre de leur maladie, de poids financier ou de lutte contre l'exclusion. Le fait qu'on ne parle pas de ces personnes en tant que «travailleurs » permet finalement de les voir plus facilement comme un «poids » ou une «menace » pour les finances publiques, plutôt que comme un apport.

\footnotetext{
${ }^{24}$ Le Conseil constitutionnel valide ainsi l'article 97 dans sa décision du 29 décembre 2003, estimant que la condition de stabilité n'était pas inconstitutionnelle à partir du moment où le dispositif des soins urgents et vitaux était créé pour pallier à ses effets. Les principes d'égalité et de protection de la santé étaient dès lors respectés.

${ }^{25}$ FASSIN, Didier, La raison humanitaire. Une histoire morale du temps présent, op. cit.

${ }^{26}$ GABARRO, Céline, L'attribution de l'aide médicale de l'Etat par les agents de l'Assurance maladie, op. cit.
} 